\title{
Electron Cryotomography
}

\author{
Gavin E. Murphy and Grant J. Jensen \\ California Institute of Technology, Pasadena, CA, USA
}

Electron cryotomography is an emerging technique that allows the structures of unique biological objects such as individual macromolecules, viruses, and even small whole cells to be reconstructed in their near-native states in three dimensions (3-D) to an approximate 5-nm resolution. The required instrumentation, sample preparation and limitations, data collection, typical results, and future prospects are summarized briefly.

\section{Introduction}

The cell is a complicated factory with ordered and regulated assembly lines whose activities are performed by macromolecular assemblies that can rightly be called machines (1). An important goal is to understand the structures and functions of these machines and then eventually manipulate them. While X-ray crystallography and nuclear magnetic resonance (NMR) spectroscopy are the most widely used tools of structural biology and can yield atomic models, they are limited to specimens that can be purified in high numbers, are conformationally homogeneous, and either crystallize or remain stable in solution in high concentrations for long periods of time. Likewise, electron cryomicroscopy-based single particle analysis techniques can generate medium-resolution $(0.5-2 \mathrm{~nm})$ reconstructions of large complexes, but again only if large numbers of structurally homogenous particles can be purified. Light microscopy can visualize unique objects, including living cells, but only to a resolution of approximately $400 \mathrm{~nm}$. Electron cryotomography promises to fill the resolution gap in between, allowing the structures of unique macromolecular machines and supramolecular assemblies like viruses, organelles, or even intact small cells to be visualized to an approximate 5-nm resolution in a near-native state (2-5).

\section{What Is ECT?}

Electron cryotomography (ECT, or alternatively cryoelectron tomography) is the combination of three core technologies. Tomography refers to a generic imaging strategy wherein a higher-dimensional reconstruction of an object is calculated from a series of lower-dimensional projections. Most people are familiar with its medical application, the computerized axial tomography (CAT) scan, where twodimensional (2-D) projections of patients are recorded with $\mathrm{X}$-rays and then a three-dimensional ( $3-\mathrm{D})$ model is calculated and analyzed on a computer. In electron tomography, electrons are used to produce the projection images in a transmission electron microscope, with far higher resolutions (appropriate for individual macromolecules) but also far less specimen penetration. The cryo prefix refers to the fact that the sample is kept cold while it is being imaged, typically after being either plunge- or high-pressure frozen. Such directly frozen samples are preserved in a more native state than the typically chemical fixed, dehydrated, resin-embedded, sectioned, and stained samples used in traditional electron microscopy, but they are also more radiation sensitive and have poorer contrast.

\section{What Is Needed to Do ECT?}

Several expensive enhancements to standard electron microscopes are needed for ECT. First, in order to keep the (vitreously) frozen water in the sample from crystallizing, stages that keep the sample cooled to approximately $-165^{\circ} \mathrm{C}$ or less are needed. The first generation of cryostages, which are still in frequent use today, have liquid nitrogen dewars attached to the end of the specimen rod. Because these require frequent refilling and suffer from drift and mechanical vibrations as the nitrogen boils away, more recently, large dewars have been mounted on the microscope and connected thermally to internal cartridges that hold the electron microscopy grids. Some of these specimen holders allow the grid to be rotated $90^{\circ} \mathrm{C}$ after one round of tilting to enable dual-axis tomography $(6,7)$. The stage goniometer must be mechanically precise and eucentric so target regions do not move out of the beam when the sample is tilted to approximately $\pm 70^{\circ} \mathrm{C}$.

Second, because inelastically scattered electrons damage the sample and do not contribute usefully to images, samples should not be much thicker than the mean inelastic free path of an electron, which for a 300-keV electron in organic materials is approximately $0.35 \mu \mathrm{m}$. Thus higherthan-normal microscope voltages (200-400 kV) are typically used to balance higher specimen penetration against losses in electron detectability. Energy filters are also used to remove lower energy, inelastically scattered electrons from the beam, which would otherwise simply add noise to the image $(8,9)$. Finally, charge-coupled device (CCD) cameras are necessary to allow real-time tracking and focusing during automatic data collection. 


\section{How Is ECT Performed?}

To prepare a sample for ECT, a drop of purified protein, virus suspension, or cell culture is typically placed upon an electron microscopy grid which is then blotted and plunged into a cryogen (10). When the sample is appropriately thin and the plunging is sufficiently fast, liquid water in the sample is frozen into vitreous ice, preserving the sample in a near-native, frozen-hydrated state. Automated plunge freezers are now available for this crucial step to improve control and reproducibility $(11,12)$, but manual blotting can still be necessary for fragile samples (13). Physiologically relevant buffers can be used $(14,15)$. Cells can be grown directly upon the grid and then frozen (16). Plunge-frozen samples are preserved so well, in fact, that enzymes, viruses, and even cells have been seen to remain active, infective, or vital after thawing.

After such a plunge-frozen sample is loaded into the microscope, target areas on the grid are identified, and a series of projection images are recorded of the sample while the grid is tilted incrementally (Figure $1 \mathrm{~A}$ ). A number of parameters must be chosen to obtain the highest resolution possible. The principal resolution-limiting factor in ECT is the dose tolerance of frozen-hydrated, biological samples: each electron has a significant probability of scattering inelastically, breaking bonds and depositing thermal energy in the sample. Thus, after even a few e-/ $\AA^{2}$, specimen damage is detectable. Nevertheless, because radiolytic fragments remain roughly in their original positions within the vitreous ice, gross information about macromolecular morphology can still be obtained through doses of up to $100 \mathrm{e}^{-} / \AA^{2}$ or more before the sample eventually bubbles, catastrophically distorting all remaining ultrastructure.

Thus if a new project were started to visualize the ultrastructure of a small bacterial cell, for instance, the dose tolerance of that particular sample would be determined by iteratively imaging it until bubbles appeared. Assuming bubbles first appeared after $150 \mathrm{e} / \AA^{2}$, the total dose used would have to be something less than that limit, perhaps $100 \mathrm{e}^{-} / \AA^{2}$. Based on previous experience, a resolution of approximately $4 \mathrm{~nm}$ might be targeted. If the thickness of the cell were $200 \mathrm{~nm}$, to reach that resolution, the tilt increment would then need to be approximately $1^{\circ} \mathrm{C}$ or finer (17). Because slab-shaped samples become too thick at high tilt angles, and the grid holder itself eventually blocks the beam, images at tilt angles greater than approximately $70^{\circ} \mathrm{C}$ cannot typically be obtained. This problem, called the missing wedge, means some information is uncollected. Assuming the goniometer and stage allow the sample to be tilted to $\pm 70^{\circ} \mathrm{C}, 141$ images would be recorded. Dividing the dose equally among the images, the average dose per image would then be approximately $0.7 \mathrm{e}^{-/} \AA^{2}$ (in fact, the dose is typically distributed unequally so that higher doses can be invested into the high tilt images, where a large fraction of the beam is inelastically scattered and removed

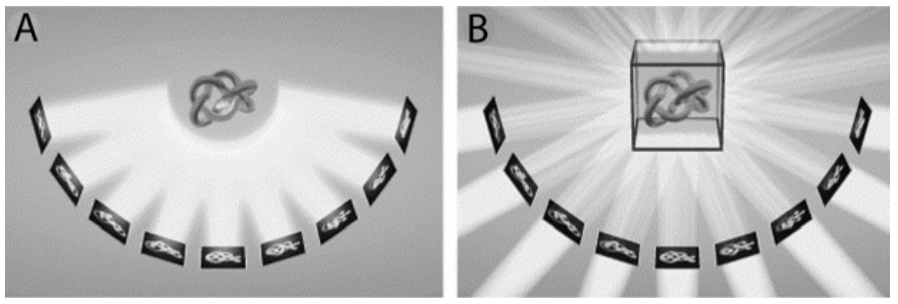

Figure 1. The principle of tomography. (A) Projections of a sample are taken from multiple angles and then (B) those images are back-projected to reconstruct the original object in three dimensions (3-D). (Reproduced from Reference 55.)

by the energy filter). The magnification would be chosen so that the pixel sampling is around one-third the desired resolution or less, or in this case approximately $1.33 \mathrm{~nm} /$ pixel. The defocus is chosen (in this case approximately $8 \mu \mathrm{m}$ underfocus) so that spatial frequencies up to and at least a little beyond the target resolution are transmitted before the first zero of the contrast transfer function, where the phase flips. This maximizes the contrast of low-resolution features while retaining information up to the target resolution (18).

Once the parameters have been chosen, the grid is positioned at the eucentric height, the focus is set, and then the target area of the grid is positioned underneath the beam using minimal dose. The tilt-series along one axis can be collected automatically using a variety of software packages such as UCSF Tomo (19), the TOM toolbox for MATLAB (20), SerialEM (21), Xplore3D (FEI Company, Eindhoven, The Netherlands), or Leginon (22). These programs incrementally tilt the sample, track its movement, deflect the beam and adjust the focus as necessary, and record an image. To collect an orthogonal, dual-axis tilt series one must remove the sample, rotate the grid $90^{\circ} \mathrm{C}$, relocate the sample, and collect a second tilt-series. This procedure minimizes the missing wedge of information.

\section{What Is the Result?}

The tilt-series are merged to produce a 3-D reconstruction, or tomogram, of the specimen. The mathematical foundations of 3-D reconstruction were developed over 40 years ago (23-26). Several algorithms exist (17), and many computer programs are available that can routinely reconstruct a data set in a few hours, including for example IMOD (27), Bsoft (28), Spider (29), Priism (30), the TOM toolbox (20), PROJALIGN (31), and Xplore3D. The most common approach is to back-project weighted images (Figure 1B) to reconstruct their 3-D forms. Initial reconstructions can then be iteratively refined against the projection images until they converge to a best-match solution. The wedge of data that is missing due to the tilt angle limitation degrades the resolution in the direction parallel to the beam. Thus horizontal features like membranes at 


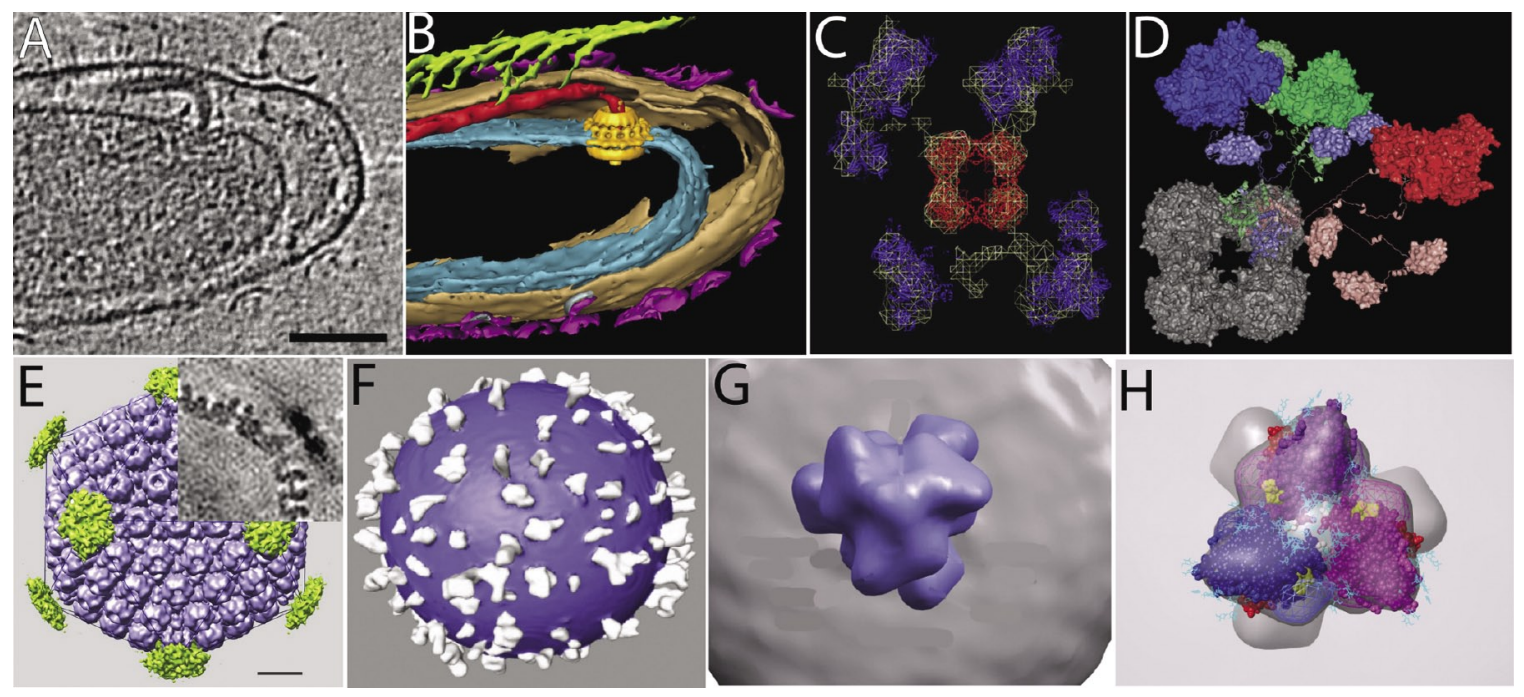

Figure 2. Examples of results produced by electron cryotomography. (A) A two-dimensional (2-D), central slice through a full three-dimensional (3-D) reconstruction of a Treponema primitia cell. Scale bar is $100 \mathrm{~nm}$. (B) A manually segmented, 3-D view of the same cell shown in panel (A) but with key components shown in different colors and the symmetrized average of many $T$. primitia flagellar motors embedded in the position of the motor. (C) A central slice through the isosurface of one pyruvate dehydrogenase multi-enzyme complex with manually fit core and subunit proteins (14). (D) A model of one corner of the same multi-enzyme complex, with three subunits flexibly tethered to the core. (E) An average of many tomographic reconstructions of Herpes virus capsids, showing the capsid proteins and portal (40). Scale bar is $20 \mathrm{~nm}$. (F) Segmented surface of an HIV capsid. (G) 3-fold symmetrized average of thousands of SIV Env spikes. (H) Crystal structures of the individual spike proteins fit into the ECT density. Panels F-H are from Reference 47.

the top and bottom of cells, for instance, are usually not resolvable.

3-D tomograms can be visually inspected, segmented, or analyzed further computationally. Volumes can be visualized as 2-D slices (Figure 2A), as 3-D surfaces, or volume-rendered using computer graphics. Using programs like IMOD (27) or Amira, the user may segment the reconstruction, which means that certain sets of voxels are assigned to various objects like membranes or macromolecular complexes. Segmented objects can then be colored differently and visualized as surfaces (Figure 2B). Manual segmentation is common, but it is subjective. Some progress has been made toward automatic or semi-automatic segmentation. For example, the user may choose a voxel, and the software will recursively add all the adjacent voxels with similar densities. Volumes, surface areas, distances, and density profiles of objects can be measured.

As a few examples, electron cryotomograms have been used to assess the relative densities and spacings of various bacterial cell wall layers. The number, relative positions, volumes, and surface areas of the various Golgi cisternae of the photosynthetic eukaryote Ostreococcus tauri have been measured (32). Cryotomograms of Magnetospirillum magneticum revealed that their magnetosomes were simply invaginations of the inner membrane organized by an actin-like cytoskeletal filament $(33,34)$. Cryotomograms of the cytoskeletal filaments in Spiroplasma melliferum suggested a mechanism for that species' mysterious mechanism of locomotion (35). As more such reconstructions are computed and loaded into databases like the Cell Centered Database (36), they will become an invaluable encyclopedia of cellular ultrastructure.

If multiple and structurally homogeneous copies of an object like a macromolecular complex are present within the tomograms, they can be computationally extracted, aligned, and averaged to produce a model with increased resolution and statistical significance. This has been performed on many structures, including the nuclear pore complex (37), microtubules $(38,39)$, surface and capsid proteins displayed by viruses (40-47) (Figure 2, E-H), the bacterial chemotactic receptor array (48), and the flagellar motor (13) (Figure 2B). Sometimes atomic structures can be fit into the lower resolution densities of subtomograms, or those produced by averaging subtomograms (Figure 2, C, G, and H). Given a template, one may also computationally search through a reconstruction to find the patterns of localization. The distribution and orientation of the ribosomes within a Spiroplasma cell were mapped in this way (49).

\section{What Is the Future of ECT?}

Both the range of samples that can be analyzed by ECT and the obtainable resolution will continue to increase. For thicker samples, such as larger bacterial cells and almost all eukaryotic cells, two strategies are being developed 
to produce slices thin enough to image. First, methods are being developed to section frozen-hydrated samples directly (cryosectioning). Unfortunately, current cryosections are usually badly compressed and are scarred by knife marks, crevasses, and rippling. Cryosections are also hard to deposit flat onto grids. Some material is inevitably lost between sections and serial sections are difficult to obtain $(50,51)$. The second strategy is to shape samples with a focused ion beam $(52,53)$. In this case, a beam of energetic gallium ions is used to etch away a carefully controlled volume of material out of a larger plunge- or high-pressure-frozen sample. To achieve higher resolutions, phase plates and spherical aberration correctors are being developed to improve the contrast of projection images, much like phase contrast objectives have done in the field of light microscopy. Direct electron detectors that perform better than film or CCD cameras should dramatically improve the quality of the images and, therefore, reconstructions (54).

In summary, ECT is becoming an increasingly valuable tool for biochemists, microbiologists, and cell biologists to determine the ultrastructures of macromolecular complexes, supramolecular assemblies, or even whole cells, and should therefore precipitate a wonderful renaissance in transmission electron microscopy.

\section{Acknowledgments}

We would like to thank Wolfgang Baumeister for allowing us to re-use Figure 1; Giovanni Cardone and Alasdair Steven for Figure 2E; and Ping Zhu, Ken Taylor and Kenneth Roux for Figure 2, F-H. This work was supported in part by NIH grants no. P01 GM66521 and R01 Al067548 to G.J.J., DOE grant no. DE-FG02-04ER63785 to G.J.J., a Searle Scholar Award to G.J.J., NIH graduate fellowship F31 EB 004179 to G.E.M., and gifts to Caltech from the Ralph M. Parsons Foundation, the Agouron Institute, and the Gordon and Betty Moore Foundation.

\section{References}

1. Alberts, B. 1998. The cell as a collection of protein machines: preparing the next generation of molecular biologists. Cell 92:291-294.

2. Jensen, G.J. and A. Briegel. 2007. How electron cryotomography is opening a new window onto prokaryotic ultrastructure. Curr. Opin. Struct. Biol. 17:260-267.

3. Lucic, V., F. Forster, and W. Baumeister. 2005. Structural studies by electron tomography: from cells to molecules. Annu. Rev. Biochem. 74:833-865.

4. McIntosh, R., D. Nicastro, and D. Mastronarde. 2005. New views of cells in 3D: an introduction to electron tomography. Trends Cell Biol. 15:43-51.

5. Subramaniam, S. and J.L. Milne. 2004. Three-dimensional electron microscopy at molecular resolution. Annu. Rev. Biophys. Biomol. Struct. 33:141-155.

6. Iancu, C.V., E.R. Wright, J. Benjamin, W.F. Tivol, D.P. Dias, G.E. Murphy, R.C. Morrison, J.B. Heymann, and G.J. Jensen. 2005. A "flip-flop" rotation stage for routine dual-axis electron cryotomography. J. Struct. Biol. 151:288-297.

7. Nickell, S., R. Hegerl, W. Baumeister, and R. Rachel. 2003. Pyrodictium cannulae enter the periplasmic space but do not enter the cytoplasm, as revealed by cryo-electron tomography. J. Struct. Biol. 141:34-42.

8. Grimm, R., M. Barmann, W. Hackl, D. Typke, E. Sackmann, and W. Baumeister. 1997. Energy filtered electron tomography of ice-embedded actin and vesicles. Biophys. J. 72:482-489.

9. Han, K.F., J.W. Sedat, and D.A. Agard. 1995. Mechanism of image formation for thick biological specimens: exit wavefront reconstruction and electron energy-loss spectroscopic imaging. J. Microsc. 178:107-119.

10. Dubochet, J., M. Adrian, J.J. Chang, J.C. Homo, J. Lepault, A.W. McDowall, and P. Schultz. 1988. Cryo-electron microscopy of vitrified specimens. Q. Rev. Biophys. 21:129-228.

11. Frederik, P.M. and D.H. Hubert. 2005. Cryoelectron microscopy of liposomes. Methods Enzymol. 391:431-448.

12. Iancu, C.V., W.F. Tivol, J.B. Schooler, D.P. Dias, G.P. Henderson, G.E. Murphy, E.R. Wright, Z. Li, et al. 2006. Electron cryotomography sample preparation using the Vitrobot. Nat. Protocols 1:2813-2819.

13. Murphy, G.E., J.R. Leadbetter, and G.J. Jensen. 2006. In situ structure of the complete Treponema primitia flagellar motor. Nature 442:1062-1064.

14. Murphy, G.E. and G.J. Jensen. 2005. Electron cryotomography of the $E$. coli pyruvate and 2-oxoglutarate dehydrogenase complexes. Structure 13:1765-1773.

15. Wagenknecht, T., R. Grassucci, J. Berkowitz, and C. Forneris. 1992. Configuration of interdomain linkers in pyruvate dehydrogenase complex of Escherichia coli as determined by cryoelectron microscopy. J. Struct. Biol. 109:70-77.

16. Medalia, O., I. Weber, A.S. Frangakis, D. Nicastro, G. Gerisch, and W. Baumeister. 2002. Macromolecular architecture in eukaryotic cells visualized by cryoelectron tomography. Science 298:1209-1213.

17. Frank, J. 2006. Electron Tomography. Springer, New York.

18. Frank, J. 2006. Three-Dimensional Electron Microscopy of Macromolecular Assemblies. Oxford University Press, New York.

19. Zheng, S.Q., B. Keszthelyi, E. Branlund, J.M. Lyle, M.B. Braunfeld, J.W. Sedat, and D.A. Agard. 2007. UCSF tomography: an integrated software suite for real-time electron microscopic tomographic data collection, alignment, and reconstruction. J. Struct. Biol. 157:138-147.

20. Nickell, S., F. Forster, A. Linaroudis, W.D. Net, F. Beck, R. Hegerl, W. Baumeister, and J.M. Plitzko. 2005. TOM software toolbox: acquisition and analysis for electron tomography. J. Struct. Biol. 149:227-234.

21. Mastronarde, D.N. 2005. Automated electron microscope tomography using robust prediction of specimen movements. J. Struct. Biol. 152:36-51.

22. Suloway, C., J. Pulokas, D. Fellmann, A. Cheng, F. Guerra, J. Quispe, S. Stagg, C.S. Potter, and B. Carragher. 2005. Automated molecular microscopy: the new Leginon system. J. Struct. Biol. 151:41-60.

23. De Rosier, D.J. and A. Klug. 1968. Reconstruction of three dimensional structures from electron micrographs. Nature 217:130-134.

24. Hart, R.G. 1968. Electron microscopy of unstained biological material: the polytropic montage. Science 159:1464-1467.

25. Hoppe, W., R. Langer, G. Knesch, and C. Poppe. 1968. Protein crystal structure analysis with electron radiation. Naturwissenschaften 55:333-336.

26. Radon, J. 1917. Über die Bestimmung von Funktionen durch ihre Integralwerte längs gewisser Mannigfaltigkeiten. Berichte 
Sächsische Akademie der Wissenschaften Math.-. Phys. KI. 69:262-267.

27. Kremer, J.R., D.N. Mastronarde, and J.R. McIntosh. 1996. Computer visualization of three-dimensional image data using IMOD. J. Struct. Biol. 116:71-76.

28. Heymann, J.B. and D.M. Belnap. 2007. Bsoft: image processing and molecular modeling for electron microscopy. J. Struct. Biol. 157:3-18.

29. Frank, J., M. Radermacher, P. Penczek, J. Zhu, Y. Li, M. Ladjadj, and A. Leith. 1996. SPIDER and WEB: processing and visualization of images in 3D electron microscopy and related fields. J. Struct. Biol. 116:190-199.

30. Chen, H., W. Clyborne, J.W. Sedat, and D.A. Agard. 1992. PRIISM: an integrated system for display and analysis of three-dimensional microscope images. Proc SPIE Int Soc Opt Eng 1660:784-790.

31. Winkler, H. and K.A. Taylor. 2006. Accurate marker-free alignment with simultaneous geometry determination and reconstruction of tilt series in electron tomography. Ultramicroscopy 106:240-254.

32. Henderson, G.P., L. Gan, and G.J. Jensen. 3-D Ultrastructure of Ostreococcus tauri: Electron cryotomography of an entire eukaryotic cell. PloS One (In press).

33. Komeili, A., Z. Li, D.K. Newman, and G.J. Jensen. 2006. Magnetosomes are cell membrane invaginations organized by the actin-like protein MamK. Science 311:242-245.

34. Scheffel, A., M. Gruska, D. Faivre, A. Linaroudis, J.M. Plitzko, and D. Schuler. 2006. An acidic protein aligns magnetosomes along a filamentous structure in magnetotactic bacteria. Nature 440:110-114.

35. Kurner, J., A.S. Frangakis, and W. Baumeister. 2005. Cryoelectron tomography reveals the cytoskeletal structure of Spiroplasma melliferum. Science 307:436-438.

36. Martone, M.E., A. Gupta, M. Wong, X. Qian, G. Sosinsky, B. Ludascher, and M.H. Ellisman. 2002. A cell-centered database for electron tomographic data. J. Struct. Biol. 138:145-155.

37. Beck, M., F. Forster, M. Ecke, J.M. Plitzko, F. Melchior, G. Gerisch, W. Baumeister, and 0. Medalia. 2004. Nuclear pore complex structure and dynamics revealed by cryoelectron tomography. Science 306:1387-1390.

38. Garvalov, B.K., B. Zuber, C. Bouchet-Marquis, M. Kudryashev, M. Gruska, M. Beck, A. Leis, F. Frischknecht, et al. 2006. Luminal particles within cellular microtubules. J. Cell Biol. 174:759765.

39. Nicastro, D., J.R. McIntosh, and W. Baumeister. 2005. 3D structure of eukaryotic flagella in a quiescent state revealed by cryo-electron tomography. Proc. Natl. Acad. Sci. USA 102:15889-15894.

40. Cardone, G., D.C. Winkler, B.L. Trus, N. Cheng, J.E. Heuser, W.W. Newcomb, J.C. Brown, and A.C. Steven. 2006. Visualization of the herpes simplex virus portal in situ by cryo-electron tomography. Virology. 361: 426-434

41. Chang, J.T., M.F. Schmid, F.J. Rixon, and W. Chiu. 2007. Electron cryotomography reveals the portal in the herpesvirus capsid. J. Virol. 81:2065-2068.

42. Deng, B., C.M. O'Connor, D.H. Kedes, and Z.H. Zhou. 2007. Direct visualization of the putative portal in the Kaposi's sarcoma-associated herpesvirus capsid by cryoelectron tomography. J. Virol. 81:3640-3644.

43. Forster, F., O. Medalia, N. Zauberman, W. Baumeister, and D. Fass. 2005. Retrovirus envelope protein complex structure in situ studied by cryo-electron tomography. Proc. Natl. Acad. Sci. USA 102:4729-4734.

44. Harris, A., G. Cardone, D.C. Winkler, J.B. Heymann, M. Brecher, J.M. White, and A.C. Steven. 2006. Influenza virus pleiomor- phy characterized by cryoelectron tomography. Proc. Natl. Acad. Sci. USA 103:19123-19127.

45. Zanetti, G., J.A. Briggs, K. Grunewald, Q.J. Sattentau, and S.D. Fuller. 2006. Cryo-electron tomographic structure of an immunodeficiency virus envelope complex in situ. PLoS Pathog 2:e83.

46. Zhu, P., E. Chertova, J. Bess, Jr., J.D. Lifson, L.O. Arthur, J. Liu, K.A. Taylor, and K.H. Roux. 2003. Electron tomography analysis of envelope glycoprotein trimers on HIV and simian immunodeficiency virus virions. Proc. Natl. Acad. Sci. USA 100:15812-15817.

47. Zhu, P., J. Liu, J. Bess, Jr., E. Chertova, J.D. Lifson, H. Grise, G.A. Ofek, K.A. Taylor, and K.H. Roux. 2006. Distribution and threedimensional structure of AIDS virus envelope spikes. Nature 441:847-852.

48. Zhang, P., R.M. Weis, P.J. Peters, and S. Subramaniam. 2007. Electron tomography of bacterial chemotaxis receptor assemblies. Methods Cell Biol. 79:373-384.

49. Ortiz, J.0., F. Forster, J. Kurner, A.A. Linaroudis, and W. Baumeister. 2006. Mapping 70S ribosomes in intact cells by cryoelectron tomography and pattern recognition. J. Struct. Biol. 156:334-341.

50. Al-Amoudi, A., J.J. Chang, A. Leforestier, A. McDowall, L.M. Salamin, L.P. Norlen, K. Richter, N.S. Blanc, et al. 2004. Cryoelectron microscopy of vitreous sections. EMBO J. 23:35833588.

51. Hsieh, C.E., A. Leith, C.A. Mannella, J. Frank, and M. Marko. 2006. Towards high-resolution three-dimensional imaging of native mammalian tissue: electron tomography of frozenhydrated rat liver sections. J. Struct. Biol. 153:1-13.

52. Marko, M., C. Hsieh, R. Schalek, J. Frank, and C. Mannella. 2007. Focused-ion-beam thinning of frozen-hydrated biological specimens for cryo-electron microscopy. Nat. Methods 4:215-217.

53. Heymann, J.A., M. Hayles, I. Gestmann, L.A. Giannuzzi, B. Lich, and S. Subramaniam. 2006. Site-specific 3D imaging of cells and tissues with a dual beam microscope. J. Struct. Biol. 155:63-73.

54. Li, S., J. Bouwer, F. Duttweiler, M. Ellisman, L. Jin, P. Leblanc, A. Milazzo, S. Peltier, et al. 2006. A new direct detection camera system for electron microscopy, p. 606800-606810. In M.M. Blouke (Ed.) Sensors, Cameras, and Systems for Scientific/ Industrial Applications VII. SPIE, San Jose, CA.

55. Baumeister, W., R. Grimm, and J. Walz. 1999. Electron tomography of molecules and cells. Trends Cell Biol. 9:81-85. 